\title{
Estrategia activo colaborativo factor influyente en el aprendizaje de Análisis Matemático I, en estudiantes de Ciencias de la Administración: Universidad Nacional del Centro del Perú - 2014
}

\section{Collaborative active strategy influential factor in learning Mathematical Analysis I, students of Management Science: National University Center \\ Peru - 2014}

\begin{abstract}
Flores Gamboa, Amelida ${ }^{1}$ / Samaniego Flores, Conny ${ }^{2}$ / Samanigo Flores, Danny ${ }^{3}$
'Facultad de Ciencias de la Administración de la Universidad Nacional del Centro del Perú

${ }^{2}$ Facultad de Ingeniería de Sistemas de la Universidad Nacional del Centro del Perú

${ }^{3}$ Centro Pre Universitario de la Universidad Nacional del Centro del Perú
\end{abstract}

\section{RESUMEN}

El presente trabajo se ha efectuado tratando de contribuir que los estudiantes mejoren en el rendimiento académico en la asignatura de Análisis Matemático. En tal sentido el objetivo general es determinar la influencia de la aplicación de la estrategia metodológica activo colaborativo en el Aprendizaje de Análisis Matemático I, en los estudiantes de Ciencias de la Administración de la Universidad $\mathrm{Na}$ cional del Centro del Perú - 2014.

El programa de Evaluación Internacional de Estudiantes, más conocido como Pisa, analiza el rendimiento de los alumnos de 15 años en asignaturas como matemáticas, lenguaje y ciencia, a partir de unas pruebas a las que fueron sometidos los escolares de 65 países, que representan el $80 \%$ de la población mundial, donde el último de la lista y del grupo de América Latina es Perú. Este problema también afecta a los estudiantes del nivel superior donde se han evidenciado: formación insuficiente en temas de matemática en la educación secundaria e inadecuadas metodologías de presentación, desarrollo y evaluación de los contenidos de las asignaturas de Matemá-

\section{ABSTRACT}

The present work has been done trying to help students improve academic performance in the course of Mathematical Analysis. In this regard, the overall objective is to determine the influence of the application of the methodological strategy collaborative active in Learning Mathematical Analysis I, students of Management Sciences, National University of Central Peru - 2014. The Program for International Student Assessment, better known as Pisa, analyzes the performance of 15 year old students in subjects such as math, language arts and science from a few tests that were subjected schoolchildren in 65 countries, representing $80 \%$ of the world population, where the last of the list and the group of Latin America is Peru. This problem also affects the upper level where students have demonstrated: insufficient training on issues of mathematics in secondary and inadequate presentation methodologies, development and evaluation of the contents of the Basic math courses in the first semester education. This research have the direct participation of 42 
tica Básica en los primeros semestres.

Esta investigación contamos con la participación directa de los 42 estudiantes del tercer semestre en su totalidad, las actividades para la aplicación fueron planeadas para el capítulo de aplicaciones de la derivada,

Finalmente se concluye que la estrategia activo colaborativo constituye el eje dinamizador con la participación activa y colaborativa de los estudiantes lo que nos permite comprobar el objetivo general planteado en la presente investigación.

Palabras Claves: Activo-colaborativo, Enfoque cuantitativo, Derivada, Pisa. students of the third semester as a whole, the activities for implementation were planned for the chapter on applications of the derivative, Finally it is concluded that the active collaborative strategy is the dynamic hub with the active and collaborative participation of students allowing us to check the overall objective in this investigation.

Keywords: active-collaborative, Quantitative Approach, Derivative, Pisa.

\section{INTRODUCCIÓN}

En la mayoría de los empleos de cualquier empresa exigen el trabajo en equipo, es decir saber trabajar con los demás integrantes de la organización, en ese escenario los docentes debemos estar conscientes de esta necesidad y tratar de incentivar el trabajo en equipo como una estrategia para desarrollar actividades sociales. Sin embargo nuestra función como docente debe ir más allá de agrupar estudiantes para desarrollar ciertas tareas como: exposiciones en grupos, trabajos en grupos, controles de lectura, etc. o dar instrucciones como: "formar grupos de trabajo para exponer tal tema" o "desarrollar la siguiente actividad en grupos", puesto que no todo trabajo en grupo en el aula es trabajo cooperativo o colaborativo. Es necesario e imprescindible enseñarle a los estudiantes alcanzar metas, cooperando unos con otros es decir aprovechar la propia experiencia y de los demás para maximizar el aprendizaje.

El aprendizaje colaborativo es un enfoque que realiza el aprendizaje entre estudiantes, da la oportunidad de enseñar y aprender en cooperación, nuestra investigación se enmarca en el aprendizaje colaborativo de la asignatura de análisis matemático el objetivo del presente trabajo es construir una estrategia metodológica activo colaborativo en el aprendizaje del aná- lisis matemático en el tema de aplicaciones de la derivada, demostrar que cuando el docente planifica adecuadamente los temas para la aplicación de la estrategia basada en el aprendizaje cooperativo o colaborativo los resultados son alentadores y queda demostrado que las matemáticas pueden ser aprendidas mediante su propio esfuerzo y el esfuerzo de los demás.

El tema del aprendizaje activo colaborativo no es nuevo pero actualmente está tomando importancia a nivel de la educación superior con mucha fuerza y buenos resultados. El docente tiene el papel protagónico, porque es el que diseña y mantiene casi por completo la estructura de las interacciones y de los resultados que se quiere obtener. El docente da instrucciones planificadas y los estudiantes se hacen cargo de su propio aprendizaje. Esta metodología es para los docentes que buscan desarrollar habilidades personales y sociales y si ponemos el esfuerzo necesario de seguro se obtiene una efectividad mayor en el proceso de aprendizaje porque se lograra ventajas en el estudiante disminuyendo los sentimientos de aislamiento y favorecer los sentimientos de autoestima. Además el trabajo en equipo como técnica didáctica, inmersa en el principio de socialización, propone capacitar a los alumnos para realizar actividades en común a fin de desarrollar la solidaridad y la cooperación. 
Finalmente Siguiendo con la idea, todo aprendizaje colaborativo, refiere la planeación previa de la clase, teniendo claros los objetivos educativos que desea lograr. El uso de estrategias de aprendizaje no convencional o tradicional, significa hacer uso del enfoque de aprendizaje constructivista en donde el estudiante pasa a ser el centro del proceso e-a (enseñanza-aprendizaje), y conlleva por lo tanto para el profesor, una mayor creatividad. Además nuestro trabajo de investigación incide como docente de la asignatura debe planificar las acciones previas a la aplicación del método en mención cuya finalidad es no solo formar simples grupos o equipos de trabajo, sino equipos de amigos que trabajan para lograr una meta del aprendizaje del tema a tratar, mediante colaboración mutua que sea más efectiva que un aprendizaje individual.

\section{MATERIALES Y MÉTODOS}

Como nuestra investigación es descriptiva explicativa con enfoque cualitativo y cuantitativo, con diseño expñortorio, pues el modelo de estrategia activo colaborativo en el tema de derivadas y sus aplicaciones, se desarrollo con una muestra intencional de 41 estudiantes pertenecientes a la única sección del tercer semestre de la facultad de Ciencias de la Administración de la UNCP, cuyo objetivo es determinar la influencia de la aplicación de la estrategia metodológica activo colaborativo en el Aprendizaje del Análisis Matemático, se utilizara el silabo de la asignatura en mención, centrándonos en los contenidos:

- Derivadas (teoría básica).

- Aplicaciones de la derivada(temas seleccionados para la aplicación de la estrategia activo colaborativo).

Se ha considerado la asignatura de Análisis Matemático del plan nuevo 2012. Incidiendo en el diagnóstico del rendimiento académico en el área de matemáticas, para después se construyo el modelo de estrategia y se procedió aplicarlo y finalmente explicar los resultados obtenidos.

\section{Población}

Todos los estudiantes matriculados en la asignatura de análisis matemático de la facultad de Ciencias de la Administración de la UNCP.

\section{Muestra}

Los estudiantes del III semestre matriculados en la asignatura de análisis matemático

1. ACOSTA MENENDOZA JAKELIN CYNTHIA $2012200001 \mathrm{~F}$

2. ALVIÑO HUAYTA KENEDY PAUL 2013200001K

3. ANCHIVILCA RODRIGUEZ 20132000031

4. ARELLANO PUCUHUARANGA DAVID AGUSTIN 2013200004H

5. AYALA SINCHI IVONNE SHEILA 2012200689L

6. CABANILLAS EGUAVIL YANINA NAYELI $2013200005 \mathrm{G}$

7. CAIRO MERCADO JULINHO JHERSON $2013200006 \mathrm{~F}$

8. CAPCHA ALVAREZ YULY MADENY $2013200007 \mathrm{E}$

9. CHAVEZ AVILA BRAYM MITCHEL 2013200008D

10. CORDOVA MELENDEZ YOEL NOEL $2013200009 \mathrm{C}$

11. CUADRADO HINOSTROZA NICOLE LUCERO $2013200010 \mathrm{~K}$

12. EGUIA PRUDENCIO MILAGROS LISSTH 2013200011

13. HIDALGO IGNACIO JHANCARLOS JUNIOR $2013200013 \mathrm{G}$

14. HUAMANI CARDENAS DALYS BEATRIZ 2010200022C

15. INIIGO TICLLACURI RAUL $2013200014 \mathrm{~F}$

16. JIMENEZ PAREJAS LISSETH ARACELY $2013200015 \mathrm{E}$

17. JORGE PINO RAIDA 2013200016D

18. LINDO RAMOS MONICA ALICIA $2013200017 \mathrm{C}$

19. MALDONADO BONIEVE RAYSA AMALIA $2013200018 B$

20. MARTINEZ HUAMAN ELVIS EFFREN $2011200394 \mathrm{G}$

21. MAYHUA SANCHEZ MAX MELVIN 2013200019A

22. MOZA MENDOZA KEVIN ROY 2013200020H

23. OCHOA GAVINO KAREN PAOLA $2013200021 \mathrm{G}$

24. ORIHUELA PRETELMELIZA LINDSAY $2012200029 \mathrm{G}$

25. PEREZ CORDOVA MONICA 2012200022F

26. QUINTANA ZEVALLOS KATTERINE ELSA 2013200024D

27. RAMIREZ ALANYA DAYSI LIDA 2013200025C

28. RAMOS RAMOS CECILIA MAXIMA 2013200026B

29. RIVEROS HUANAY MAX WILLIAMS 2013200027A

30. RODRIGUEZ AGUILAR ERICK DANIEL 2013200028L

31, RODRIGUEZ QUISPE IRAIDA 2012200845F

32. ROJAS POMAHUALI ENMA DABIDA 2013200029K

33. ROMAN CORDOVA ESTEFANI CAROLINE $2013200030 \mathrm{~F}$

34. ROMERO VILCAPOMA RAUL 2013200031E

35. SEDANO CUADROS SONIA 2013200032D

36. TADEO OTOS LAWRENCIA CRISTINA $2013200033 \mathrm{C}$

37. TAIPE MAURATE MILAGROS PILAR 2013200034B

38. VARGAS OSORIO ESTELA MARA 2013200035A

39. VELIZ MUÑOZ KENNYI MARTIN 2010200608A

40. VERASTEGUI QUIPE ANTHONI SIMON 2013200036L

41. VIVANCO ENRIQUEZ GABY GISELA 2013200037K 
Tabla 1. Población de estudio

\begin{tabular}{lcccccc}
\hline SECCION & \multicolumn{3}{c}{$\begin{array}{c}\text { ESTUDIANTES } \\
\text { SEXO }\end{array}$} & \multicolumn{2}{c}{ TOTAL } \\
\hline III SEMESTRE & \multicolumn{3}{c}{$M$} & \multicolumn{3}{c}{$\mathrm{F}$} \\
SECCIÓN “A" & $\mathrm{N}^{\circ}$ & $\%$ & $\mathrm{~N}^{\circ}$ & $\%$ & $\mathrm{~N}^{\circ}$ & $\%$ \\
& 16 & 39 & 25 & 61 & 41 & 100 \\
TOTAL & 16 & 39 & 25 & 61 & 41 & 100 \\
\hline
\end{tabular}

Fuente: elaboración propia- Archivo FCA

\section{RESULTADOS}

1. Para medir resultados la influencia de la estrategia activo colaborativo: se realizo un seguimiento del rendimiento académico de los estudiantes matriculados en el III semestre de la FCA - 2014 II; cuyos resultados son como sigue:

Tabla 2. Notas de matemática I - 2013-I

\begin{tabular}{|c|c|c|}
\hline Notas & Cantidad & Porcentaje \% \\
\hline $13-14$ & 5 & 14 \\
\hline $15-16$ & 28 & 80 \\
\hline $17-18$ & 2 & 6 \\
\hline Total & 35 & $100 \%$ \\
\hline
\end{tabular}

La tabla $N^{\circ} 2$ nos muestra como ha sido el rendimiento académico de los 35 estudiantes al culminar el I semestre, el resultado es satisfactorio puesto que no hay desaprobados la nota más alta es 18 en la asignatura de matemática I.

Tabla 3. Notas de matemática II - 2014-I

\begin{tabular}{|c|c|c|c|}
\hline Notas & Cantidad & Porcentaje & $\%$ \\
\hline $06-07$ & 3 & 7 & \\
\hline $08-10$ & 6 & 15 & \\
\hline $11-13$ & 29 & 71 & \\
\hline $14-15$ & 3 & 7 & \\
\hline Total & 41 & $100 \%$ & \\
\hline
\end{tabular}

Fuente: Elaboración propia; Archivo FCA
La tabla $\mathrm{N}^{\circ} 3$ nos muestra como ha sido el rendimiento académico de los 41 estudiantes al culminar el I semestre 2014, el resultado no es satisfactorio puesto que hay nueve estudiantes desaprobados y la nota más alta es 15 en la asignatura de matemática II.

Tabla 4. Notas de análisis matemático 1er consolidado del III semestre-2014 II

\begin{tabular}{ccc}
\hline Notas & Cantidad & Porcentaje \\
\hline $05-07$ & 7 & 17 \\
$08-10$ & 16 & 39 \\
$11-13$ & 11 & 27 \\
$14-16$ & 7 & 17 \\
Total & 41 & $100 \%$ \\
\hline
\end{tabular}

Fuente: Elaboración propia; Archivo FCA

La tabla $\mathrm{N}^{\circ} 4$ nos muestra el rendimiento académico de los 41 estudiantes al primer consolidado del 2014-II, el resultado no es satisfactorio puesto que hay 23 estudiantes desaprobados en la asignatura de análisis matemático. Inicialmente se tuvo 30 desaprobados con una segunda oportunidad lograron aprobar 7 estudiantes mas.

2. Con los resultados de las tablas $N^{\circ} 2,3$, y 4 ; se toma la decisión de modelar la estrategia activo colaborativo para ser aplicada antes del 2 do consolidado, para lo cual se tuvo en consideración el contenido de silabo de análisis matemático II , 2014 - II, centrándonos en las semanas $N^{\circ} 7$ al $N^{\circ} 11$. 


\section{CONTENIDOS Y CRONOGRAMA}

Tabla 5.

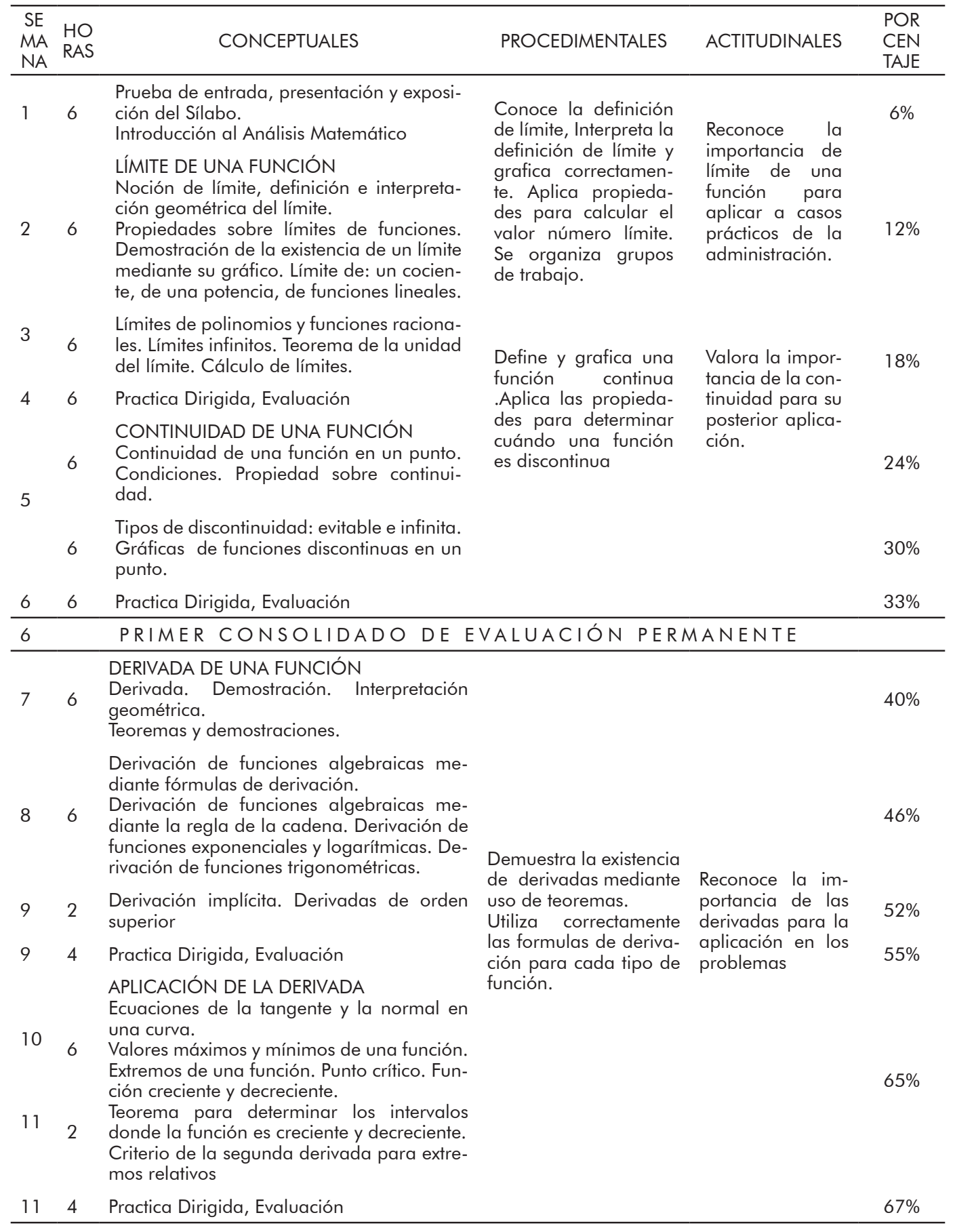




\begin{tabular}{|c|c|c|c|c|}
\hline 11 & & CONSOLIDADO DE & EVALUACIÓN PERMANENTE & \\
\hline 12 & 6 & $\begin{array}{l}\text { INTEGRAL INDEFINIDA Y APLICACIONES } \\
\text { Diferencial, interpretación geométrica de la } \\
\text { diferencial. La anti derivada de una función }\end{array}$ & & $75 \%$ \\
\hline 13 & 6 & $\begin{array}{l}\text { Integral indefinida, definición, notación, } \\
\text { teoremas } \\
\text { Integrales y fórmulas de integración inme- } \\
\text { diatas } \\
\text { Formulas básicas de integración y Integra- } \\
\text { ción por sustitución }\end{array}$ & & $80 \%$ \\
\hline 14 & 2 & $\begin{array}{l}\text { Métodos y Técnicas de Integración, Integra- } \\
\text { ción de funciones trigonométricas, Integra- } \\
\text { ción por partes, Integración de funciones } \\
\text { racionales. }\end{array}$ & $\begin{array}{l}\text { Reconoce, utiliza y re- } \text { Valora la im- } \\
\text { suelve problemas de su portancia de la } \\
\text { entorno con cada una aplicación de de- } \\
\text { de las formulas de de- }\end{array}$ & $85 \%$ \\
\hline 14 & 4 & Practica Dirigida, Evaluación & rivación y aplicaciones gocios. & \\
\hline 15 & 6 & $\begin{array}{l}\text { INTEGRAL DEFINIDA, ÁREAS Y APLICACIO- } \\
\text { NES } \\
\text { Integral definida. Propiedades } \\
\text { Teorema fundamental del cálculo integral }\end{array}$ & & \\
\hline 15 & & $\begin{array}{l}\text { Calculo de integral definida usando interva- } \\
\text { los de igual longitud } \\
\text { Áreas de regiones planas, Áreas bajo la } \\
\text { curva }\end{array}$ & & \\
\hline 16 & 6 & Practica Dirigida, Evaluación & & $95 \%$ \\
\hline 17 & 6 & SEMANA DE LA CALIDAD UNIVERSITARIA & & $100 \%$ \\
\hline 17 & & CONSOLIDADO DE & EVALUACIÓN PERMANENTE & $100 \%$ \\
\hline
\end{tabular}

3. Se diseñó y se aplicó la estrategia activo colaborativo, con modificaciones de la técnica de rompecabezas, especialmente para la asignatura de análisis matemático, obteniendo el siguiente resultado al culminar el segundo consolidado:

Tabla 6. Notas de análisis matemático 2 do consolidado del III semestre-2014 II

\begin{tabular}{ccccc}
\hline Notas & Cantidad & Porcentaje & $\%$ \\
\hline $05-07$ & 2 & 5 & \\
$08-10$ & 0 & 0 & \\
$11-\quad 13$ & 12 & 29 & \\
$14-\quad 16$ & 27 & 66 & \\
Total & 41 & 100 & $\%$ \\
\hline
\end{tabular}

Fuente: Elaboración propia; Archivo FCA

La tabla 5 nos muestra como ha sido el rendimiento académico de los 41 estudiantes después de aplicar la estrategia activo colaborativo, este resultado es en el segundo consolidado del III semestre 2014-II, el resultado es satisfactorio puesto que hay solo dos estudiantes desaprobados en la asignatura de análisis matemático. Asimismo, no solo el resultado es alentador de tener solo dos estudiantes desaprobados como lo muestra la tabla $N^{\circ} 5$, sino que lo principal es haber logrado que los grupos formados al azar, donde no estaban amigos y amigas que se frecuentan constantemente, hayan logrado una amistad socio afectiva y una cohesión extraordinaria que les permitió formar un equipo de trabajo colaborativo, demostrando responsabilidad, ayuda, solidaridad, respeto y sentimientos de obligatoriedad.

Al finalizar esta experiencia se aplicó un cuestionario a los estudiantes del III semestre (Anexo $N^{\circ} 4$ ) para conocer la satisfacción de todo este proceso.

\section{DISCUSIÓN}

Con la seguridad de los resultados podemos mencionar: 
* Para enseñar estrategias no solo de enseñanza sino también de aprendizaje, la responsabilidad de éxito es compartida con el estudiante y en especial del docente como se muestra el siguiente esquema:

Figura 1.

\section{ENSEÑAR ESTRATEGIAS DE APRENDIZAJE}

\begin{abstract}
Significa para nuestros estudiantes
Enseñarles a reflexionar sobre su propia manera de aprender, ayudándoles a analizar las operaciones y decisiones mentales que realizan, con el fin de mejorar los procesos cognitivos que ponen en acción
\end{abstract}

Enseñarles a conocerse mejor, a identificar sus dificultades, habilidades y preferencias en el momento de aprender y ayudarles a construir su "propia identidad cognitiva"

Enseñarles a dialogar internamente, activando sus conocimientos previos sobre los contenidos a tratar y relacionándolos con la nueva información.

Enseñarles a ser intencionales y propositivos cuando aprendan y responder a las intenciones y las demandas de sus profesores y de los demás

Enseñarles, que no deben estudiar para aprobar sino para aprender, que lo que se aprende es fruto de un esfuerzo de comprensión y resulta más duradero y funcional.
Pasos para estructurar el proceso de Enseñanza - aprendizaje mediante la Estrategia Activo Colaborativo. Si bien es cierto estos pasos se considero para la modelación de la estrategia activo colaborativo, cuya actividad tiene como finalidad que los partici-

\begin{tabular}{l}
\hline Supone para nosotros, docentes \\
\hline Reflexionar sobre nuestra propia manera de \\
planificar, presentar y evaluar los distintos \\
contenidos del área o sub-área que enseña- \\
mos
\end{tabular}

Reconstruir conscientemente nuestros significados como "enseñantes" con respeto a qué es lo que debe o no enseñarse; supone un reconocimiento de nuestras habilidades y carencias como profesores que nos impulsa a emprender cambios que mejoren nuestra actuación profesional

Facilitarles la tarea, explicándoles nuestras intensiones educativas, lo que conlleva un proceso preliminar de auto-reflexión con el fin de clarificarlas.

Aprender a enseñar mejor esforzándonos a comprender los motivos que nos impulsan a tomar decisiones mientras realizamos una sesión de clase. pantes, reunidos en grupos y aplicando la técnica del rompecabezas puedan exponer e intercambiar sus ideas sobre las características del aprendizaje colaborativo en el entorno del ambiente universitario. 
Figura 2.

PASOS

1. Especificar objetivos de enseñanza

2. Decidir el tamaño del grupo.

3. Asignar estudiantes a los grupos.

4. Preparar o condicionar el aula.

5. Planear los materiales de enseñanza.

6. Asignar los roles para asegurar la interdependencia.

7. Explicar las tareas académicas

8. Estructurar la meta grupal de interdependencia positiva.

9. Estructurar la valoración individual.

* Las principales dificultades que afrontamos para aplicar la estrategia activo colaborativo fueron:

- Es la que para formar los grupos de trabajo tuvimos que romper el mito que solo puedo trabajar con mis amigos y amigas de mi entorno más cercano.

- No todo grupo de trabajo es un grupo de aprendizaje cooperativo.

- Los docentes y estudiantes continúan con el criterio de los grupos de trabajo tradicionales donde algunos estudiantes habilidosos son lo que asumen un liderazgo y solo ellos se benefician de la experiencia a expensas de los miembros menos habilidosos. Solo algunos son los que trabajan académicamente y otros cubren funciones de apoyo (fotocopiado o digitando etc.).

* Como docente al aplicar la estrategia se trabajo igual o más que el estudiante si se quiere alcanzar los objetivos:

- Al tratarse de una asignatura del área de matemáticas se seleccionó con sumo cuidado los temas considerados en el sílabo, con los cuales trabajaron los estudiantes en sus respectivos grupos.

- Se debe dar énfasis en la motivación de la aplicación de la estrategia.
10. Estructurar la cooperación intergrupo.

11. Explicar los criterios del éxito.

12. Especificar las conductas deseadas.

13. Monitorear la conducta de los estudiantes.

14. Proporcionar asistencia con relación a la tarea.

15. Intervenir para enseñar con relación a la tarea.

16. Proporcionar un cierre a la lección.

17. Evaluar la calidad y cantidad de aprendizaje de los alumnos

18. Valorar el funcionamiento del grupo

- Explicar con claridad los propósitos de la asignatura o lección.

- Formar los grupos al azar.

- Explicar con claridad a los estudiantes la tarea y la estructura de meta.

- Se monitoreo la efectividad de los grupos durante todo el proceso.

- Evaluar el nivel de logros de los alumnos y ayudarles a discutir, que también hay que colaborar unos a otros.

Aportes a la modelación de la estrategia para temas matemáticos:

- Se tuvo en cuenta durante todo el proceso el silabo de la asignatura.

- Asesoramiento del docente durante todo el proceso.

- Evaluación permanente en cada etapa del proceso.

- Se modifico la técnica de rompecabezas para adecuación a los temas seleccionados de la asignatura de análisis matemático.

- Se formó los grupos por sorteo, creando una sana expectativa. 4 estudiantes por grupo.

- Presentación de los grupos cumpliendo requisitos (diseñar y describir el logo del grupo, libre presentación de cada uno de los miembros). 
- Explicación y entrega de los temas(4) a cada grupo (Anexo $\mathrm{N}^{\circ}$ ).

- Decisión del grupo para la distribución de los temas. Cada miembro se convierte en especialista del tema asignado.

- Asesoramiento de parte del docente acerca del contenido de cada tema, para todos los miembros de cada grupo.

- Conformación del grupo de especialistas por cada tema.

- Cada especialista regresa a su grupo y comparte toda información de su tema, mediante una exposición evaluada por el docente. Cada integrante debe utilizar medios y materiales creativos para su exposición.

- Entrega de material por parte de cada integrante según el tema asignado.

- Finalmente evaluación escrita a todos los estudiantes de los 4 temas.

\section{CONCLUSIONES}

1. Los estudiantes del tercer semestre de la FCA manifiestan que la estrategia activo colaborativo ha contribuido con el aprendizaje de los temas respecto a las aplicaciones de la derivada de una función.

2. Las modificaciones a la técnica del rompecabezas de la estrategia activo colaborativo es recomendable para el área de matemática.

3. Los estudiantes y docentes aún siguen trabajando con grupos de trabajo tradicionales.

4. El buen uso de la estrategia activo colaborativo influye en forma significativa en el aprendizaje de análisis matemático.

5. La motivación en el aula depende de la interacción entre el profesor y sus estudiantes.

6. El papel de las distintas estrategias de aprendizaje tienen como meta desafiante en el proceso educativo que el aprendizaje sea capaz de actuar en forma autónoma y especialmente en grupos colaborativos.

7. El principal responsable de la tarea evolutiva en el aula debe ser el docente.

8. El docente responsable debe tener dominio y experiencia en la asignatura, para seleccionar los temas para aplicar las estrategias correspondientes.

9. El papel de las distintas estrategias de aprendizaje tienen como meta desafiante en el proceso educativo que el aprendizaje sea capaz de actuar en forma autónoma y autorregulada.

\section{RECOMENDACIONES}

1. El verdadero docente debe poner en práctica las diferentes estrategias que le permitan hacer del aprendizaje significativo logros éxitos en beneficios de los aprendices en todo el proceso de enseñanza aprendizaje.

2. El profesor debe poseer un cierto conocimiento teórico y práctico más o menos preciso de todo un nutrido arsenal de instrumentos y técnicas para evaluar los aprendizajes de los alumnos.

3. Activar y generar conocimientos previos mediante la motivación y la presentación de objetivos y el uso de diferentes tipos de estrategia de enseñanza.

4. Los docentes deben actualizarse en la aplicación de estrategias de enseñanza y aprendizaje.

5. Los docentes cuando aplican nuevas estrategias en determinadas asignaturas debemos estar actualizados en estrategias para orientar la atención de los estudiantes para mantener la atención de los aprendices durante una clase. Son de tipo construccional pueden darse de manera continua para indicar a los alumnos que las ideas deben centrar sus procesos de atención codificación y aprendizaje.

6. Es necesario promover la colaboración y el trabajo grupal, ya que este establece mejores relaciones con los demás estudiantes, aprenden más, les agrada pertenecer grupo, se sienten más motivados, aumenta su autoestima y aprenden habilidades sociales más efectivas al hacer en grupos cooperativos.

7. El docente conocedor de su tarea y manejando bien el aprendizaje cooperativo con ese tratamiento constructivista, dando autonomía a sus alumnos es casi seguro lograr éxito en el aprendizaje. 


\section{REFERENCIAS BIBLIOGRÁFICAS}

1. Hernández Sampiere, Roberto; Fernández Collado, Carlos; Baptista Lucio, Pilar. (2014). Metodología de la investigación. Edición. Editorial Mc Graw Hill. México.

2. Bernal Torres, Cesar Augusto. (2010). Metodología de la Investigación, para Administración, Economía, humanidades y Ciencias Sociales. Editorial Pearson, Colombia.

3. Ñaupas Paitan, Humberto; Mejía Mejía, Elías; Novoa Ramírez, Eliana; Villagómez Paucar, Alberto. (2014). Edición de la U. Colombia.

4. Bunge, Mario. (1972). La Investigación Científica (su Estrategia y Filosofía), 2da Edición Ariel, Barcelona - España.

5. Caballero Romero, Alejandro E. (2009). Innovaciones en las guías metodológicas para los planes y tesis de maestría y doctorado, Editorial Instituto Metodológico Alen Caro Lima.

6. Méndez Álvarez, Carlos Eduardo. (2013). Metodología Diseño y desarrollo del proceso de Investigación con énfasis en Ciencias Empresariales. Edición. Editorial Limusa. México.

7. Tafur Portilla, Raúl; Izaguirre Sotomayor, Manuel. (2014). Cómo hacer un proyecto de Investigación. Edición. Lima Perú.

8. Tamayo y Tamayo, Mario. (2013). Diccionario de la Investigación Científica. Edición. Editorial Limusa. Colombia.

9. Tokeshi Shirota, Alberto. (2013). Planifique, desarrolle y Apruebe su Tesis. Universidad de Lima. Fondo editorial.

10. Lee, Clare. (201 1). El Lenguaje en el Aprendizaje de las Matemáticas. Ed. Madrid Morata.

11. Castillo Arredondo, S. (2006). Formación del profesorado en la Educación Superior. Ed. Mac Graw Hill, España.

12. Moreno Rizo, Héctor. (1999). Evaluación del Docente Universitario una Visión Institucional. Universidad Autónoma de Occidente Cali Colombia.

13. Constantino Domínguez. (1999). Tesis "El desempeño Docente, Metodologías Didácticas y el Rendimiento de los alumnos de la Escuela Académico Profesional de Obstetricia de la Facultad de Medicina".
14. Landa Fitzgerald, Victoria; Técnicas Activas y Colaborativas. Magis PUCP. Lima. (2009)

15. Bernardo Carrasco, J. (1995). Cómo Aprender mejor, Estrategias de Aprendizajes. Rialp. Madrid.

16. Jones, F.; Palincsar, A. (1995). Estrategias para enseñar a aprender. AIQUE. Buenos Aires.

17. Nisbet, J. y Shucksmith, J. (1987). Estrategias de aprendizaje. Editorial Siglo. Madrid.

18. Selmes, I. (1988). La mejora de las Habilidades para el Estudio. Paidos. Madrid. 